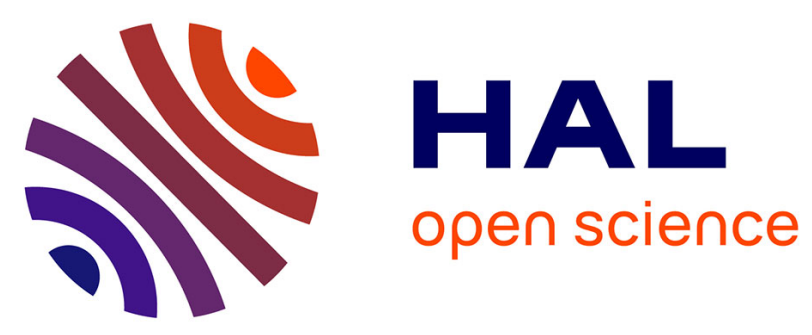

\title{
Nrf2-heme oxygenase- 1 axis in mucoepidermoid carcinoma of the lung: Antitumoral effects associated with down-regulation of matrix metalloproteinases
}

Magdalena Tertil, Slawomir Golda, Klaudia Skrzypek, Urszula Florczyk, Kazimierz Weglarczyk, Jerzy Kotlinowski, Monika Maleszewska, Szymon Czauderna, Chantal Pichon, Claudine Kieda, et al.

\section{To cite this version:}

Magdalena Tertil, Slawomir Golda, Klaudia Skrzypek, Urszula Florczyk, Kazimierz Weglarczyk, et al.. Nrf2-heme oxygenase-1 axis in mucoepidermoid carcinoma of the lung: Antitumoral effects associated with down-regulation of matrix metalloproteinases. Free Radical Biology and Medicine, 2015, 89, pp.147-157. 10.1016/j.freeradbiomed.2015.08.004 . hal-01657418

\author{
HAL Id: hal-01657418 \\ https://hal.science/hal-01657418
}

Submitted on 10 Feb 2018

HAL is a multi-disciplinary open access archive for the deposit and dissemination of scientific research documents, whether they are published or not. The documents may come from teaching and research institutions in France or abroad, or from public or private research centers.
L'archive ouverte pluridisciplinaire HAL, est destinée au dépôt et à la diffusion de documents scientifiques de niveau recherche, publiés ou non, émanant des établissements d'enseignement et de recherche français ou étrangers, des laboratoires publics ou privés. 


\title{
Nrf2-heme oxygenase-1 axis in mucoepidermoid carcinoma of the lung: Antitumoral effects associated with down-regulation of matrix metalloproteinases
}

\author{
Magdalena Tertil ${ }^{\mathrm{a}, \mathrm{b}, \mathrm{c}}$, Slawomir Golda ${ }^{\mathrm{a}, \mathrm{c}}$, Klaudia Skrzypek ${ }^{\mathrm{a}, \mathrm{b}, 1}$, Urszula Florczyk ${ }^{\mathrm{a}}$, \\ Kazimierz Weglarczyk ${ }^{\text {b,2 }}$, Jerzy Kotlinowski ${ }^{\mathrm{a}, 3}$, Monika Maleszewska ${ }^{\mathrm{a}, 4}$, \\ Szymon Czauderna ${ }^{\mathrm{a}}$, Chantal Pichon ${ }^{\mathrm{b}}$, Claudine Kieda ${ }^{\mathrm{b}, \mathrm{d}}$, Alicja Jozkowicz ${ }^{\mathrm{a}}$, \\ Jozef Dulak ${ }^{\mathrm{a}, \mathrm{d}, *}$ \\ ${ }^{a}$ Department of Medical Biotechnology, Faculty of Biochemistry, Biophysics and Biotechnology, Jagiellonian University, Gronostajowa 7, 30-387 Krakow, \\ Poland \\ ${ }^{\text {b } C e n t r e ~ d e ~ B i o p h y s i q u e ~ M o l e c u l a i r e, ~ C N R S ~ U P R ~ 4301, ~ R u e ~ C h a r l e s ~ S a d r o n ~} 45071$ Cedex 2 Orléans, France \\ c Department of Molecular Neuropharmacology, Institute of Pharmacology Polish Academy of Sciences, Smetna 12, 31-343 Krakow, Poland \\ ${ }^{\mathrm{d}}$ Malopolska Centre of Biotechnology, Jagiellonian University, Gronostajowa 7A, 30-387 Krakow, Poland
}

Lung mucoepidermoid carcinoma (MEC) is a very poorly characterized rare subtype of non-small-cell lung cancer (NSCLC) associated with more favorable prognoses than other forms of intrathoracic ma-lignancies. We have previously identified that heme oxygenase-1 (HO-1, encoded by HMOX1) inhibits MEC tumor growth and modulates the transcriptome of microRNAs. Here we investigate the role of a major upstream regulator of HO-1 and a master regulator of cellular antioxidant responses, transcription factor Nrf2, in MEC biology. Nrf2 overexpression in the NCI-H292 MEC cell line mimicked the phenotype of HO-1 overexpressing cells, leading to inhibition of cell proliferation and migration and down-reg-ulation of oncogenic miR-378. HMOX1 silencing identified HO-1 as a major mediator of Nrf2 action. Nrf2-and HO-1 overexpressing cells exhibited strongly diminished expression of multiple matrix metallo-proteinases and inflammatory cytokine interleukin-1 $\beta$, which was confirmed in an NCI-HO- 1 xenograft model. Overexpression of HO- 1 altered not only human MMP levels in tumor cells but also murine MMP levels within tumor microenvironment and metastatic niche. This could possibly contribute to decreased metastasis to the lungs and inhibitory effects of HO-1 on MEC tumor growth. Our profound tran-scriptome analysis and molecular characterization of the mucoepidermoid lung carcinoma helps to understand the specific clinical presentations of these tumors, emphasizing a unique antitumoral role of the Nrf2-HO-1 axis.

Keywords: Mucoepidermoid carcinoma, Non-small cell lung cancer, Nrf2, Nuclear factor (erythroid-derived 2)-like 2 Heme oxygenase-1, Matrix metalloproteinases, MMP-1

\footnotetext{
* Corresponding author at: Department of Medical Biotechnology, Faculty of Biochemistry, Biophysics and Biotechnology, Jagiellonian University, Gronostajowa 7, 30-387 Krakow, Poland; fax: +48 126646918.

E-mail address: jozef.dulak@uj.edu.pl (J. Dulak).

${ }^{1}$ Current affiliation: Department of Transplantation, Polish-American Institute of Pediatrics, Jagiellonian University Medical College, Krakow, Poland

${ }^{2}$ Current affiliation: Department of Clinical Immunology, Polish-American Institute of Pediatrics, Jagiellonian University Medical College, Krakow, Poland

${ }^{3}$ Current affiliation: Department of General Biochemistry, Faculty of Biochemistry, Biophysics and Biotechnology, Jagiellonian University, Krakow, Poland

${ }^{4}$ Current affiliation: Max Planck Research Group Chromatin and Ageing, Max Planck Institute for Biology of Ageing, Cologne, Germany
}

\section{Introduction}

Nrf2 (nuclear factor erythroid 2-related factor 2, encoded by NFE2L2) is a redox-sensitive transcription factor driving major molecular responses to protect cells against oxidative and electrophilic stress. Under physiological conditions, Nrf2 is sequestered within the cytoplasm while bound to its negative regulator Keap-1 (Kelch-like erythroid-derived cap-n-collar homology-associated protein-1), which serves as an adapter for cullin-3-dependent E3 ubiquitin ligase directing Nrf2 for proteasomal degradation. Upon stress, the Nrf2-Keap-1 complex is disrupted through modifications of key sensory cysteine residues of Keap-1 by reactive oxygen species or electrophiles [1]. In the nucleus, Nrf2 
dimerizes with small Maf protein and binds to the antioxidant response element (ARE) or electrophile response element (EpRE) to transactivate transcription of a broad array of cytoprotective genes, including phase 2 detoxifying enzymes and antioxidant genes such as $\mathrm{NAD}(\mathrm{P}) \mathrm{H}$ :quinone oxidoreductase-1 (NQO-1), glutathione S-transferase (GST), and heme oxygenase-1 (HO-1), an inducible isoform of the enzyme that degrades pro-oxidant heme into biliverdin, ferrous iron, and carbon monoxide.

Nrf2 plays a dual role in tumor growth and its action is largely context-dependent. On one hand, regarding tumor initiation, Nrf2 deficiency has been associated with increased susceptibility to carcinogenesis in multiple models and thus the transcription factor is a central target for chemoprevention $[2,3]$. On the other, aberrant Nrf2 activation enhances tumorigenic potential, not only through its cytoprotective effects, but also by actively promoting cancer cell proliferation, angiogenesis, and metastasis [4]. Similar oncogenic properties have been attributed to the principal Nrf2 target protein $\mathrm{HO}-1$ in many tumor types [5]. In lung cancers, the neoplasms causing the largest number of cancer-related deaths worldwide, Nrf2 gain-of-function and/or Keap1 loss-of-function mutations often occur [6]. Accordingly, high intratumoral levels of Nrf2 and HO-1 have been associated with poor clinical outcomes in most common types of lung tumors [7,8]. Nevertheless, we have recently identified a particular subtype of non-small-cell lung carcinoma (NSCLC) where HO-1 acts rather as a tumor suppressor, inhibiting cancer cell proliferation, migration, tumor growth, and angiogenesis [9].

Mucoepidermoid carcinoma (MEC) of the lung is a very rare form of intrathoracic malignancy originating from the submucosal bronchial glands and affecting approximately $0.2 \%$ of lung cancer patients [10]. Interestingly, this subtype is associated with more favorable prognoses than other NSCLCs, as the MEC tumors usually have lower grades and are diagnosed at lower clinical stages, allowing more effective surgical procedures [11]. Yet very little is known about the microenvironment and molecular characteristics of this subtype. Besides our recent profiling of microRNAs in the $\mathrm{NCI}-\mathrm{H} 292$ MEC cell line overexpressing HMOX1 [9], there is one clinical study showing that low-grade pulmonary MECs are characterized by significantly attenuated expression of the matrix metalloproteinases (MMPs) MMP-2 and MMP-9 in comparison to typical lung cancer [12]. MMPs are proteolytic enzymes degrading extracellular matrix components, which play an important role in tumor growth and progression [13]. Accordingly, we found that the expression levels of MMP-2 were associated with tumorigenic potential in $\mathrm{NCI}-\mathrm{H} 292$ xenografts [9], indicating that the regulation of MMPs may be of importance for the growth of MEC.

Here we investigate the molecular consequences of activation of the Nrf2-HO-1 axis in a model of human mucoepidermoid carcinoma of the lung. We show its atypical antitumoral actions in this subtype of NSCLC and identify the down-regulation of MMPs as one of the potential mechanisms contributing to the phenotype.

\section{Materials and methods}

\subsection{Ethics statement}

All experiments were carried out in accordance with good animal practice and were approved by the I Local Ethical Committee for Animal Research at the Jagiellonian University, Krakow.

\subsection{Cell culture}

The human NSCLC cell lines NCI-H292 (mucoepidermoid carcinoma, purchased from ATCC), A549 (adenocarcinoma, purchased from ATCC), and NCI-H460 (large cell carcinoma, purchased from
ATCC) were cultured in RPMI 1640 (PAA), and SK-MES-1 (squamous cell carcinoma, purchased from ATCC) was cultured in MEM (Gibco), each supplemented with 10\% fetal bovine serum (PAA) and penicillin $(100 \mathrm{U} / \mathrm{mL}) /$ streptomycin $(10 \mu \mathrm{g} / \mathrm{mL})$ (Sigma) (pen/ strep) under standard culture conditions: $37^{\circ} \mathrm{C}, 5 \% \mathrm{CO}_{2}, 95 \%$ humidity. NCI-H292 cell lines overexpressing NFE2L2 or HMOX1 were developed earlier in our laboratory as described in [14] and [9], respectively.

\subsection{Measurement of Nrf2 transcriptional activity}

Cells were transfected with ARE-Luc plasmid using Lipofectamine2000 (Invitrogen) according to the vendor's protocol, and Luc activity was measured as described earlier [15].

\subsection{Measurement of cell proliferation and migration in vitro}

Cell proliferation was determined using BrdU incorporation assay (cell proliferation ELISA, Roche) according to the manufacturer's protocol. A scratch assay for measurement of cell migration was performed as in [14].

\subsection{Assay of ALDH activity}

Cells were collected during trypsinization and stained using an ALDEFLUOR Kit (StemCell Technologies) according to the vendor's protocol. Flow cytometric analysis was performed on BD LSR II (the cytometry platform of the CBM UPR4301, CNRS, Orléans, France).

\subsection{Quantitative RT-PCR}

RNA was isolated using QIAzol (Qiagen) reagent according to the manufacturer's instructions and reverse-transcribed into cDNA using a RevertAid Premium First Strand cDNA Synthesis Kit (Fermentas) and nCodeVilo (Invitrogen) for analysis of miRNA. Realtime PCR was performed using QuantiTect SYBR Green (Qiagen) or SYBR Green JumpStart Taq Ready Mix (Sigma) on a Light Cycler 480 II (Roche) or a StepOne Plus (Applied Biosystems) platform. Gene expression was calculated according to delta Ct or delta delta Ct methods with EF2 and U6 as reference genes for mRNA and miRNA analysis, respectively.

\subsection{SiRNA transfection}

Cells were seeded in 24-well plates at low confluency (30-50\%) and transfected with $50 \mathrm{nM}$ chemically modified siRNA targeted at HO-1 (Stealth RNAi HMOX1 siRNA, Invitrogen) or control siRNA (Stealth RNAi Negative Control siRNA, Invitrogen) using $1 \mu \mathrm{l} /$ well of Lipofectamine2000 transfection reagent (Invitrogen) according to the protocol of the supplier.

\subsection{Microarray gene expression profiling}

NCI-H292 cells, control and overexpressing HMOX1, were cultured for $24 \mathrm{~h}$ in serum-deprived medium after reaching confluence. Then the cells were washed with PBS and lysed in QIAzol Lysis Reagent (Qiagen) and RNA was isolated by a modified Chomczynski method following the manufacturer's recommendations. The RNA was quantified using a NanoDrop-1000 spectrophotometer and quality was monitored with an Agilent 2100 Bioanalyzer (Agilent Technologies, Santa Clara, CA). RNA was hybridized to Agilent Whole Human Genome Oligo Microarrays (G4112F) and changes of expression of at least 1.5 -fold $(p<0.05)$ were considered significant. GoMiner software was used for segregation of the genes into ontology groups. Datasets are available 
through GEO (accession number GSE22030).

\subsection{Protein expression analysis}

Western blot for Nrf2 and HO-1 was performed as described earlier [16].

ELISA for IL-1 $\beta$ (R\&D Systems) was performed on cell culture media according to the vendor's protocol.

\subsection{MMPs activity measurement}

Total activity of matrix metalloproteinases was estimated using a SensoLyte 520 Generic MMP Assay Kit (AnaSpec), according to the vendor's instructions.

\subsection{In vivo tumor growth}

Xenografting of NCI-ctrl and NCI-HO-1 cells has been described earlier [9]. Briefly, 8-week-old NOD SCID mice (males) were injected subcutaneously with $5 \times 10^{6} \mathrm{NCI}-\mathrm{H} 292$ control or HMOX1 overexpressing cells in $100 \mu \mathrm{l}$ of PBS, and animals were sacrificed 7 to 8 weeks after xenografting and the serum, tumor, and lung samples were collected for further analysis.

\subsection{Immunofluorescent staining}

For immunofluorescent staining for PCNA (proliferation) samples were incubated overnight at $4{ }^{\circ} \mathrm{C}$ with anti-human PCNA antibodies (Dako, dilution 1:200) and secondary Alexa 488-conjugated antibodies (Invitrogen, dilution 1:200). Slides were mounted in a Vectashield medium (Vector Labs) containing DAPI dye. Samples were photographed using a Nikon Eclipse TS100 microscope equipped with a Nikon Digital Sight DS-5 M camera. Images were analyzed with GSA Image Analyzer software.

\subsection{Screening for inflammatory cytokines in blood}

Concentration of human cytokines in mouse serum was evaluated with BD Cytometric Bead Array (Becton Dickinson) according to the manufacturer's protocol. The results were validated with appropriate ELISA assays.

\subsection{Statistical analysis}

Results are presented as mean \pm SEM. Unpaired two-tailed Student's $t$-tests were used to assess whether the means of two groups differed significantly. For comparison of multiple groups, one-way ANOVA analysis with Bonferroni's post-test was employed.

\section{Results}

3.1. Establishment of NCI-H292 cell line with stable overexpression of $\mathrm{Nrf} 2$

To investigate the action of Nrf2 in lung mucoepidermoid carcinoma cells, NCI-H292 cell line was engineered to overexpress the transcription factor. Human NFE2L2 cDNA was stably integrated into NSCLC cells by means of retroviral infection and selection of transduced cells. The resulting NCI-Nrf2 cell line exhibited elevated Nrf2 expression and activity, as evidenced using western blotting and ARE-Luc reporter construct (Fig. 1A, 1B). Increased Nrf2 activity was further confirmed by the expression of $\mathrm{Nrf} 2$ target genes, including $\mathrm{NAD}(\mathrm{P}) \mathrm{H}$ dehydrogenase, quinone 1 (NQO1), glutamate-cysteinyl ligase catalytic domain (GCLC), and glutathione S-transferase (GST), which were uniformly up-regulated in NCI-Nrf2 cells (Fig. 1C). Accordingly, NCI-Nrf2 cells exhibited enhanced resistance to oxidative stress (data not shown). The levels of HO-1 mRNA and protein were also significantly elevated (Figs. 1A, 1C). On the other hand, Nrf2 regulatory protein Keap-1
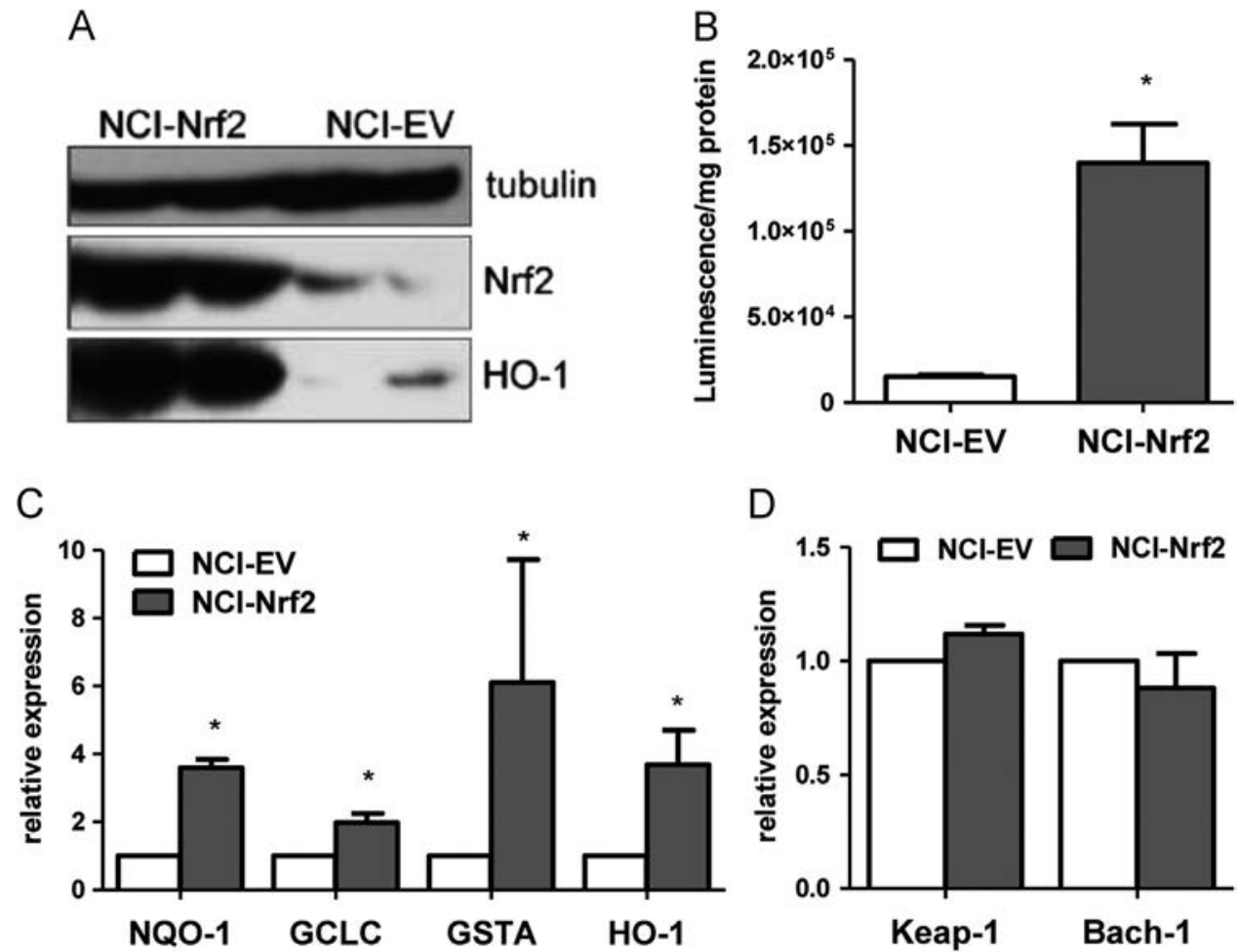

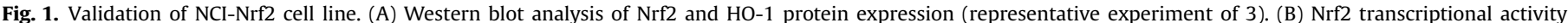
determined by ARE-Luc assay $(n=2)$. (C-D) qPCR analysis of mRNA expression of Nrf2 target genes (C) and negative regulators (D) ( $n=4)$. 

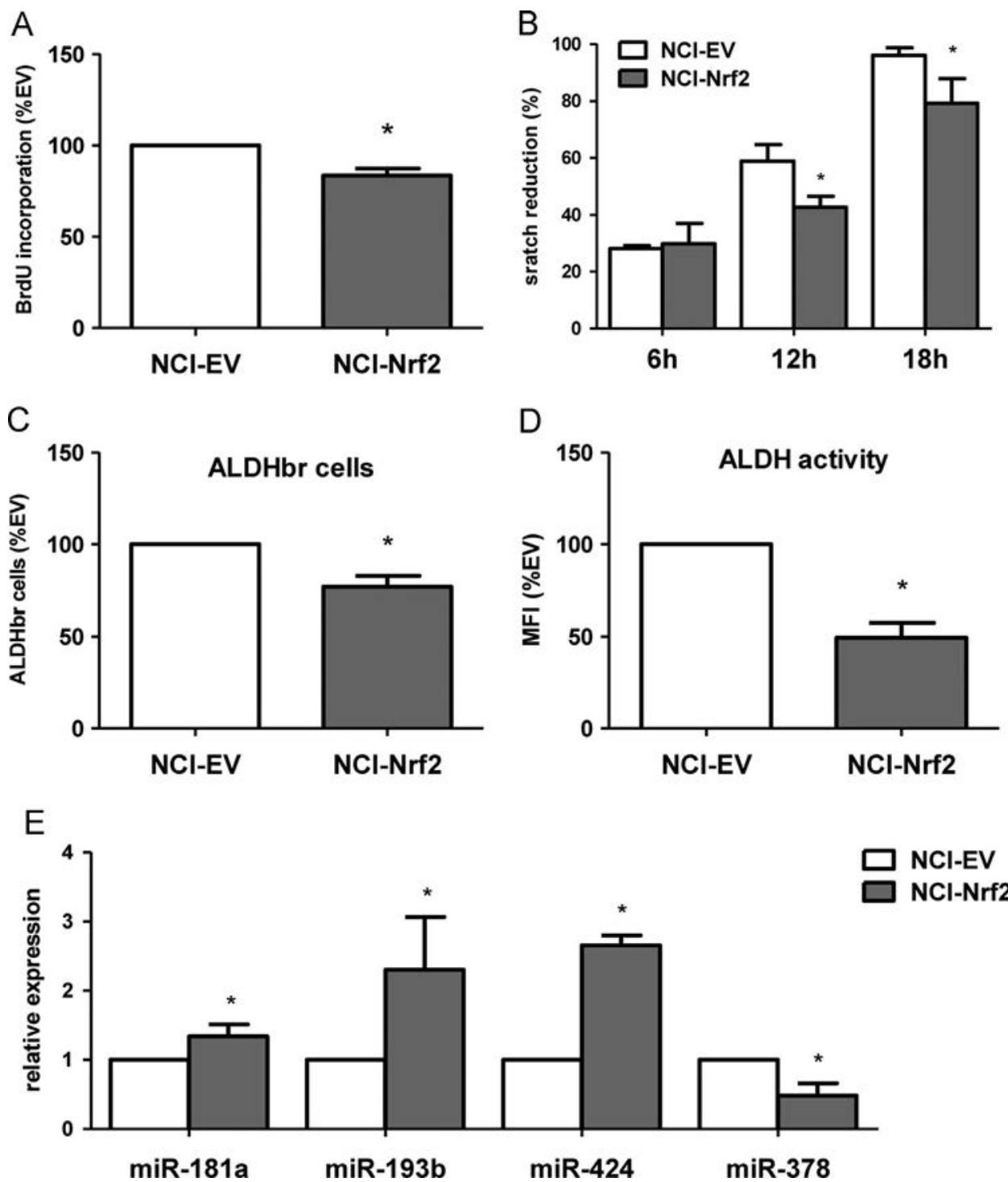

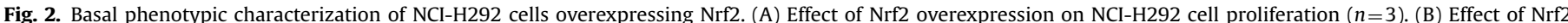

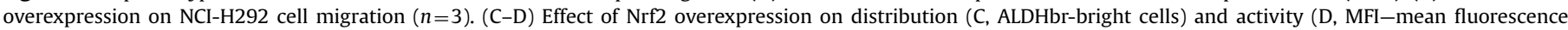

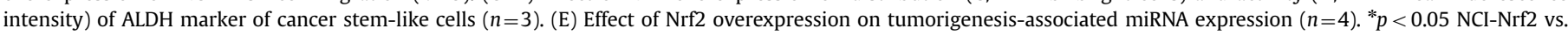
NCI-EV.

and Bach-1, which compete for binding ARE sequences, were unaffected (Fig. 1D), indicating that Nrf2 overexpression did not lead to a compensatory regulation of negative modulators of its activity.

\subsection{Nrf2 overexpression alters the phenotype of NCI-H292 cells in} vitro in a manner similar to HMOX1 overexpression

First we investigated the effect of Nrf2 overexpression on basic characteristics of the NCI-H292 cell line. NCI-Nrf2 cells exhibited attenuated proliferation and migration in vitro (Fig. $2 \mathrm{~A}, 2 \mathrm{~B}$ ). These effects were similar to the phenotype of the NCI-H292 cell line overexpressing HMOX1 [9]. Moreover, activity of ALDH, an established marker of cancer stem-like cells in non-small-cell lung carcinoma, was also diminished in Nrf2-overexpressing cells (Figs. 2C, 2D). As we have previously established that the attenuation of tumorigenic potential of HMOX1-overexpressing NCI$\mathrm{H} 292$ cells could be associated with an altered expression of microRNAs [9], we next measured the abundance of selected previously identified miRs in our model. Tumor suppressor microRNAs miR-181a, miR-193b, and miR-424 were significantly upregulated in Nrf2-overexpressing cells, while oncogenic miR-378 was down-regulated (Fig. 2E), again resembling the profile of the NCI-HO-1 cell line [9]. Altogether, these data suggested that HO-1 could be an important mediator of $\mathrm{Nrf} 2$ action in our model of lung mucoepidermoid carcinoma.

\subsection{HMOX1 knockdown reverses the major effects of Nrf2 over- expression in $\mathrm{NCI}-\mathrm{H} 292$ cells}

The involvement of HO- 1 in the action of Nrf2 in the NCI-H292 line was determined by knocking down its expression by siRNA transfection. Targeted down-regulation of HMOX1 in NCI-Nrf2 cells to the basal level of control NCI-EV cells (Fig. 3A) resulted in enhanced cell proliferation and an increased migration rate (Figs. 3B, 3C), partially and completely reversing the effects of Nrf2 overexpression, respectively. Moreover, HMOX1 knockdown in Nrf2overexpressing cells led to up-regulation of miR-378 (Fig. 3D), which plays an important role in NCI-292 tumorigenesis [9]. miR181a and miR-424 were unaffected (Figs. S1A, S1B), while miR193b was up-regulated in both the control and the NCI-Nrf2 cells (Fig. S1C) treated with siHO1, which suggests an unknown potential mechanism of $\mathrm{HO}-1$ influence in regulation of this 

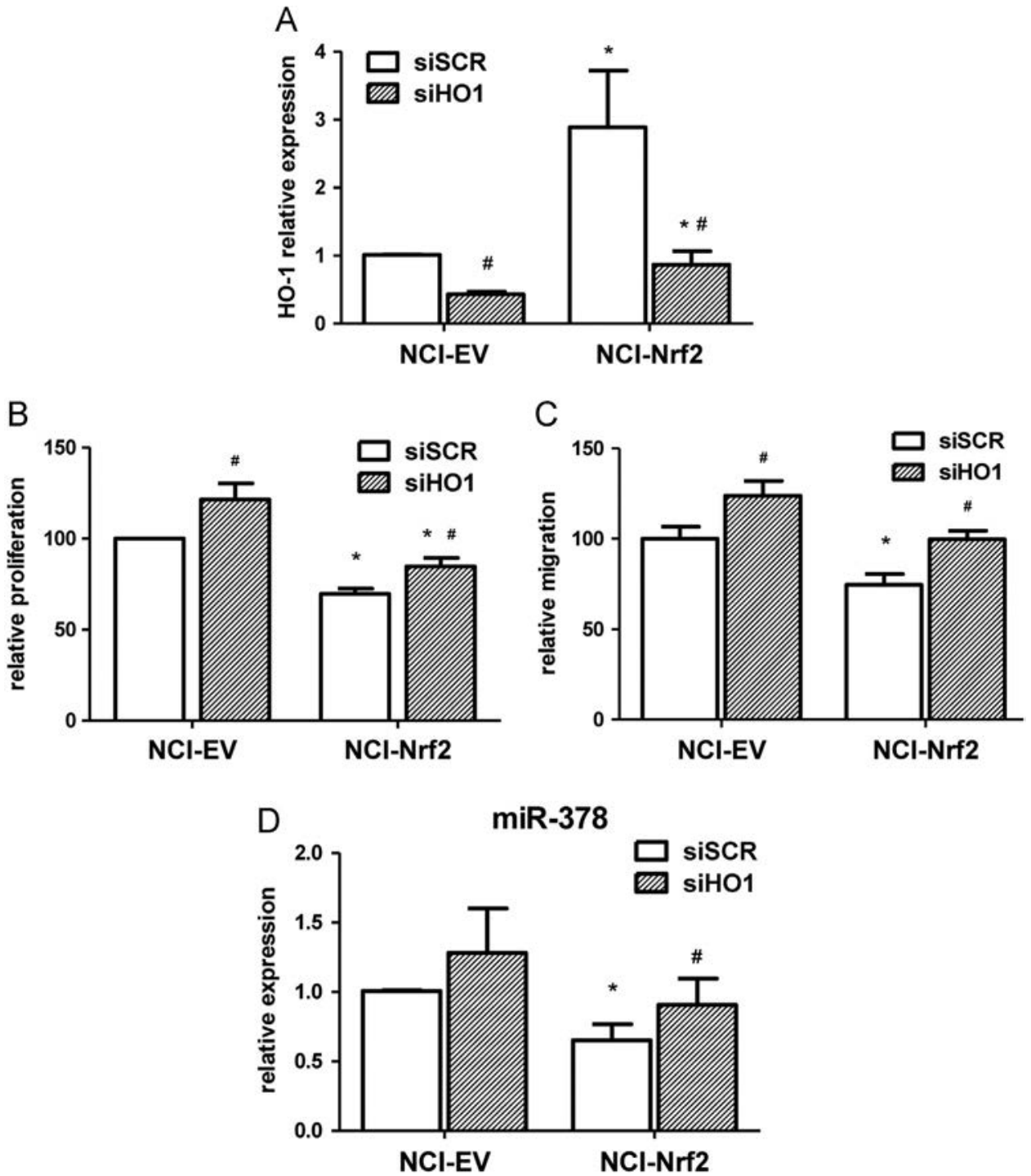

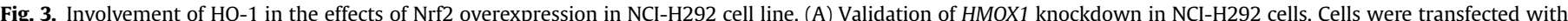

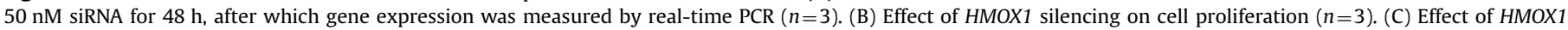

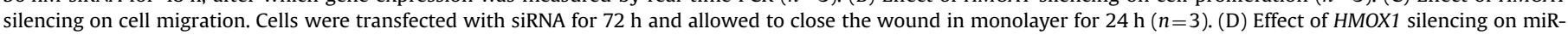
378 expression $(n=3) .{ }^{*} p<0.05$ NCI-EV vs. NCI-Nrf2, $\# p<0.05$ siSCR vs. siHO1.

microRNA.

The reversal of Nrf2-overexpression-related attenuation of cell proliferation, migration, and miR-378 expression in NCI-H292 cells by HMOX1 knockdown indicates that $\mathrm{HO}-1$ is an important downstream mediator of action of Nrf2 in this type of NSCLC.

\subsection{Attenuation of tumorigenic potential of NCI-H292 cells over-} expressing HMOX1 is associated with modulation of the mRNA transcriptome

Since HO- 1 emerged as the key player in the antioxidant response of NCI-H292 NSCLC cells, we next focused on the mechanisms underlying the antitumor effects of $\mathrm{HO}-1$ in this cell type. We chose to use the model of HMOX1 overexpression by stable plasmid transfection developed earlier in our laboratory [9], as it closely resembled the moderate HO-1 up-regulation observed in NCI-Nrf2 cells. We have already shown diminished tumor growth of the NCI-HO-1 cell line in vivo [9]. Post mortem analyses confirmed the attenuation of tumorigenic potential of NSCLC cells exerted by the enzyme: NCI-HO- 1 tumor cell proliferation and metastasis to lungs were severely inhibited (Fig. 4A, B).
To better understand the molecular changes involved in the effects of HMOX1 overexpression in our model of lung mucoepidermoid carcinoma, we performed microarray analysis of mRNA transcriptome of control and $\mathrm{NCI}-\mathrm{HO}-1$ cells cultured in vitro. We detected a variety of significant alterations in expression levels of genes regulating cell cycle, apoptosis, angiogenesis, cytokine production, and tumor metastasis, which are presented in a heat map (Fig. 4C) and in Supplementary Table 1. We found that expression of cyclins D1 and D2 (CCND1 and CCND2) was down-regulated in HMOX1 overexpressing cells (over 3.5-fold), while the genes known to inhibit cell proliferation, such as growth arrest-specific 1 (GAS1) and cyclin-dependent kinase inhibitor $1 \mathrm{C}$ (CDKN1C), were very strongly up-regulated (46- and 7-fold, respectively). The analysis demonstrated inhibition of the expression of inflammatory cytokines such as IL-1 $\alpha$ (12-fold), IL-1 $\beta$ (17-fold), IL-8 (over 200-fold), and proangiogenic growth factor VEGF-A (almost 2-fold) in cells overexpressing HMOX1. Proapoptotic caspase-9 (CASP9) and RUNX3 were induced and the mRNA level of MDM2, a negative regulator of p53, was diminished. Cells with elevated expression of HMOX1 also displayed a lower level of urokinase plasminogen activator (PLAU, 6-fold) and matrix metalloproteinase-1 (MMP1, over 20-fold), 


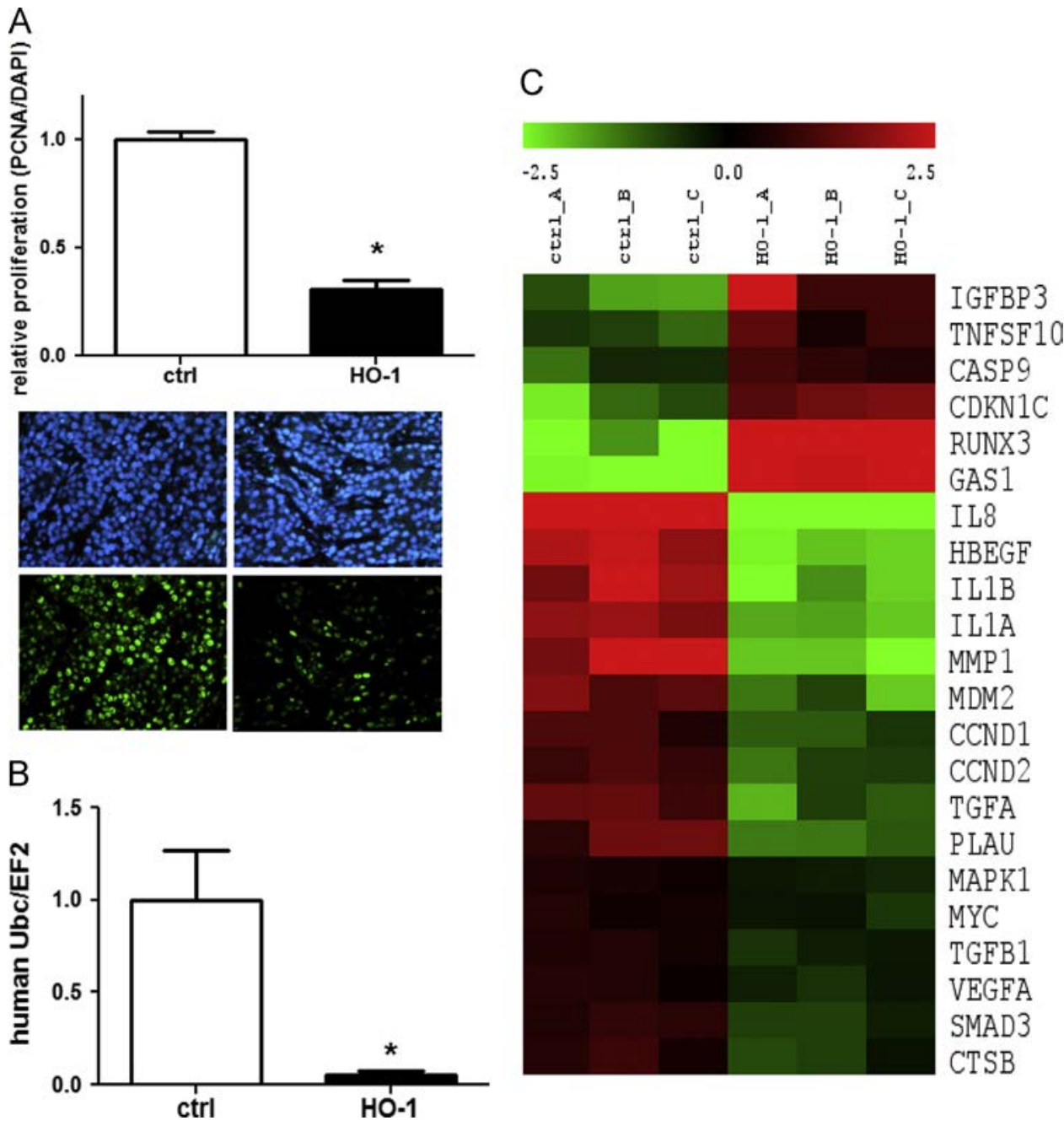

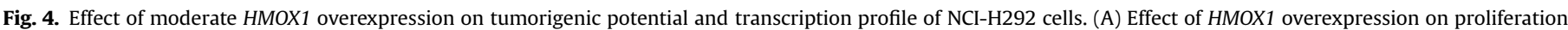

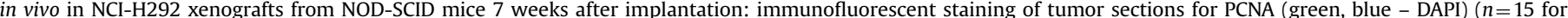

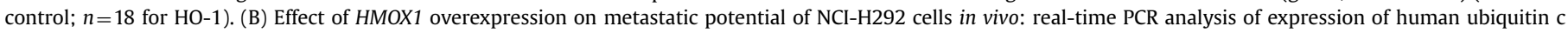

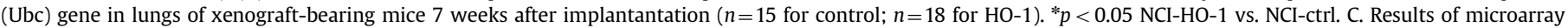
analysis of gene expression in control and HO-1-overexpressing $\mathrm{NCI}-\mathrm{H} 292$ cells in vitro.

which could contribute to the decreased metastatic potential of tumor cells.

\subsection{Nrf2-HO-1 axis regulates matrix metalloproteinases in NCI- H292 lung carcinoma}

Out of the multiple genes regulated by HMOX1 overexpression in NCI-H292 cells identified by microarray analysis, our attention was drawn to MMP-1, which has a well-established role as a negative prognostic factor in human lung cancer [17] and was potently down-regulated in NCI-HO-1 cells (Fig. 5A). Real-time PCR analysis additionally revealed inhibition of expression of MMP-9 and MMP-12 in HMOX1-overexpressing cells (Fig. 5A), which was corroborated by a significant attenuation of total MMP activity in cell culture media (Fig. 5B), suggesting that HO-1 could be an important regulator of MMPs. The striking down-regulation of MMP-1 and the regulation of MMP-9 was retained in NCI-HO-1 tumors in vivo, where diminished expression of MMP-13 and upregulation of tissue inhibitors of the MMPs (TIMPs) TIMP-3 and TIMP-4 were also identified (Figs. 5C, 5D), which, altogether, could have contributed to the inhibition of xenograft growth and metastasis. Interestingly, we observed a similarly strong attenuation of MMP-1 expression in Nrf2-overexpressing cells (Fig. 5E). It was accompanied by a down-regulation of several other MMPs, including MMP-3, MMP-12, and MMP-13, which, together with MMP-1, form a gene cluster on chromosome 11q22.3, suggesting that there may be a common regulatory mechanism partially related to HO-1. Indeed, siRNA-mediated silencing of HMOX1 in NCINrf2 cells reversed the down-regulation of MMP-3 and MMP-12 (Fig. 6B, 6D) and led to the partial restoration of MMP-1 (Fig. 6A). Additionally, a striking induction of MMP-1 and MMP-3 as well as an up-regulation of MMP-12 and MMP-9 was observed in the control cell line with HMOX1 knockdown (Figs. 6A-6D), where the expression level of the enzyme was lowest (Fig. 3A), further validating $\mathrm{HO}-1$ as a strong modulator of MMP expression in MEC. Involvement of HO-1 in the regulation of expression of MMP-1 was identified also in NCI-H460 large cell carcinoma cells, while HMOX1 silencing had no effect on MMP-1 mRNA levels in A549 lung adenocarcinoma and SK-MES1 squamous cell carcinoma cell lines (Fig. 6E), showing high specificity of HO-1 action for the NSLCLC subtype.

3.6. Nrf2 and HO-1 modulate expression of inflammatory cytokines in NCI-H292 cells

A recent report by Petrella et al. described interleukin $1 \beta$ as a key factor involved in regulation of expression of MMP-1 and 
A

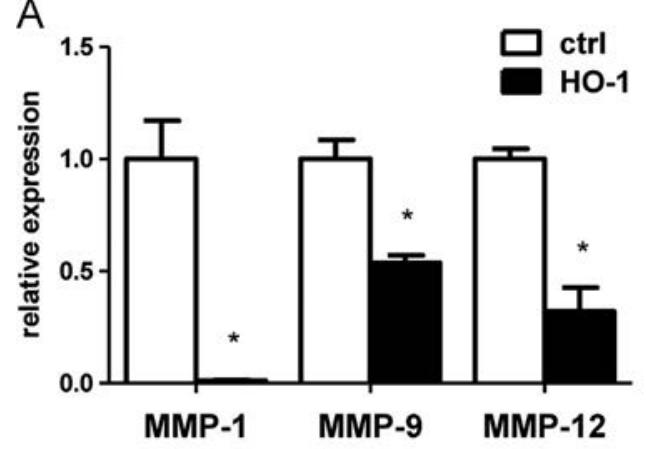

C

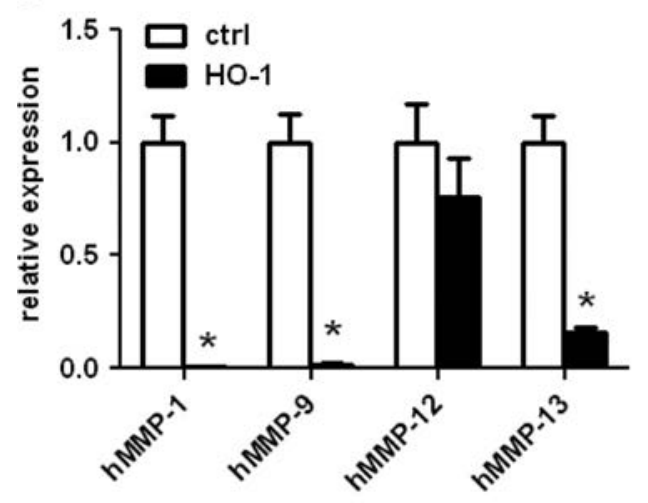

E

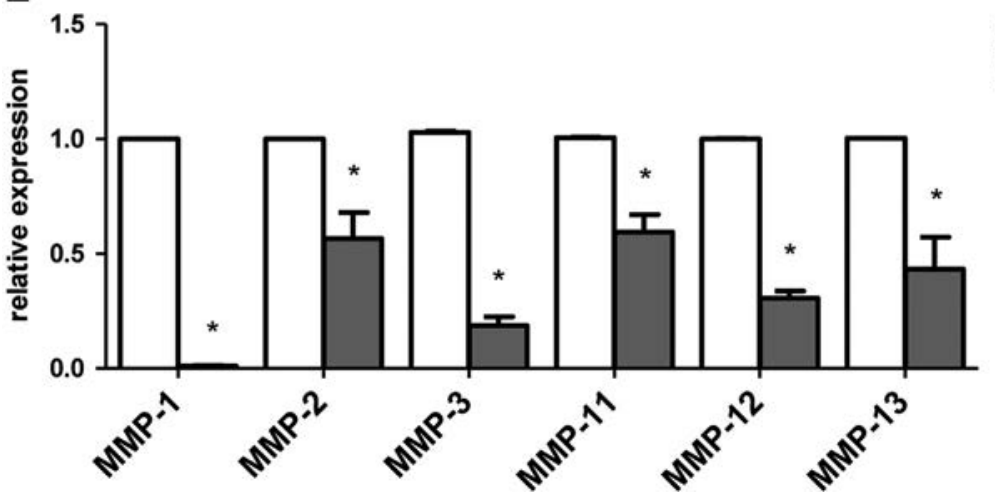

B
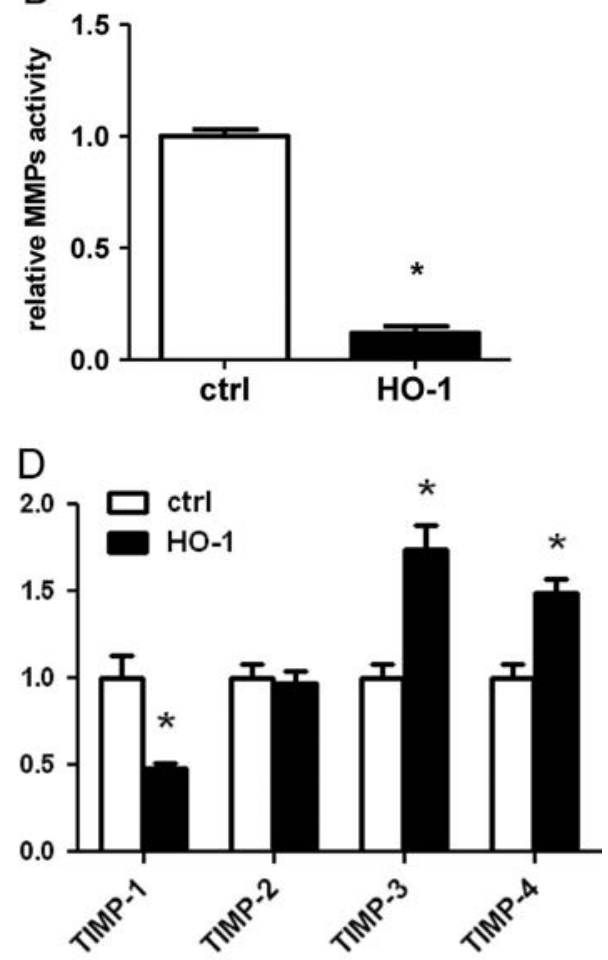

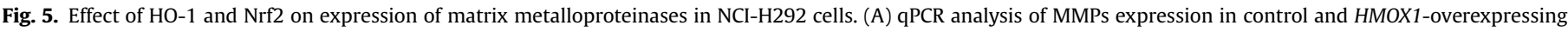

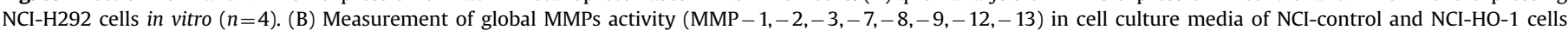

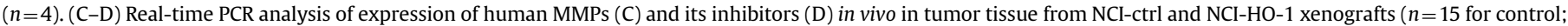
$n=18$ for HO-1). (E) qPCR analysis of MMPs expression in control and Nrf2-overexpressing NCI-H292 cells in vitro ( $n=4$ ). * $p<0.05$ NCI-EV/ctrl vs. NCI-transgene.

MMP-3 in A549 lung adenocarcinoma cells [18]. Our microarray analysis indicated that this cytokine was strongly down-regulated in HMOX1-overexpressing mucoepidermoid carcinoma cells, which was confirmed by measurement of factor production in cell culture media (Fig. 7A). Also, Nrf2-overexpressing cells exhibited diminished expression of IL-1 $\beta$, suggesting a possible association with HO-1 (Fig. 7B). Yet silencing of HMOX1 in NCI-Nrf2 cells did not alter IL-1 $\beta$ expression (Fig. 7B), showing that different pathways might be involved in $\mathrm{HO}-1$-dependent regulation of the MMPs in Nrf2-overexpressing cells. On the other hand, a twofold up-regulation of IL-1 $\beta$ in control cells treated with siRNA against HMOX1 was observed, which could have contributed to the corresponding induction of MMP $-1,-3,-9$, and -12 . (Figs. 6A$6 \mathrm{D})$. The involvement of HO- 1 in the regulation of IL-1 $\beta$, however, was specific for MEC cells, as HMOX1 silencing in cell lines of other NSCLC subtypes did not significantly change IL-1 $\beta$ expression (Fig. 7C).

Importantly, the attenuation of expression of IL- $1 \beta$ by HO- 1 in
MEC cells was confirmed in xenografts (Fig. 7D). Significant downregulation of interleukin-6 (IL-6) and tumor necrosis factor $\alpha$ (TNF $\alpha$ ) was also observed in vivo in NCI-HO-1 tumors (Fig. 7D), consistent with the known anti-inflammatory actions of the HO-1 enzyme in other cell types. Decreased levels of human IL-6 were noticed in murine serum (Fig. 7E), while human IL-1 $\beta$ and TNF $\alpha$ levels were undetectable. The altered microenvironment of HMOX1-overexpressing tumors could have affected stromal cells and metastatic niches, as we observed lower expression of murine MMP-9 in tumors and lungs of NCI-HO-1 xenograft-bearing mice (Fig. 7F).

\section{Discussion}

The salient finding of the present study is that, contrary to what has been demonstrated for the most common types of NSCLC $[19,20]$, in mucoepidermoid carcinoma the activation of 
A

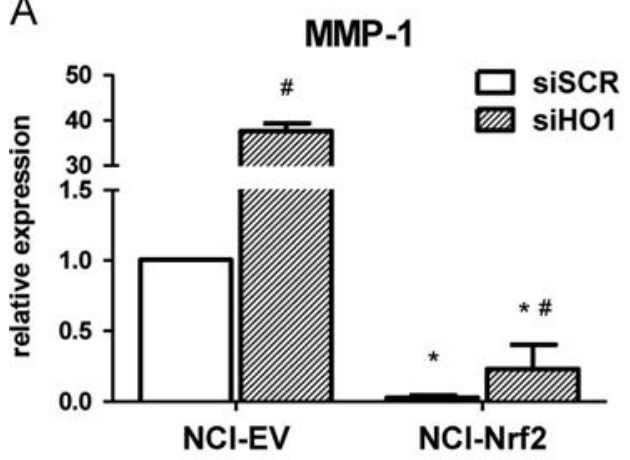

C

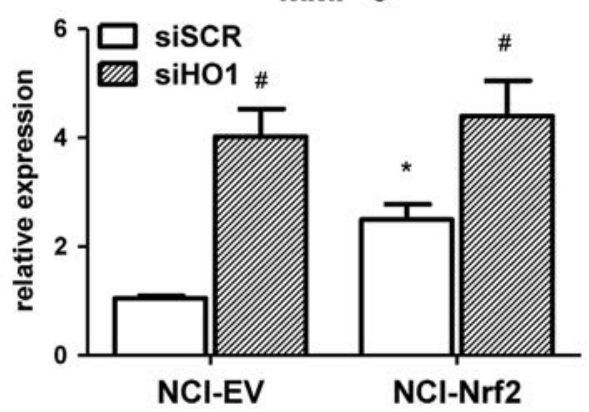

E

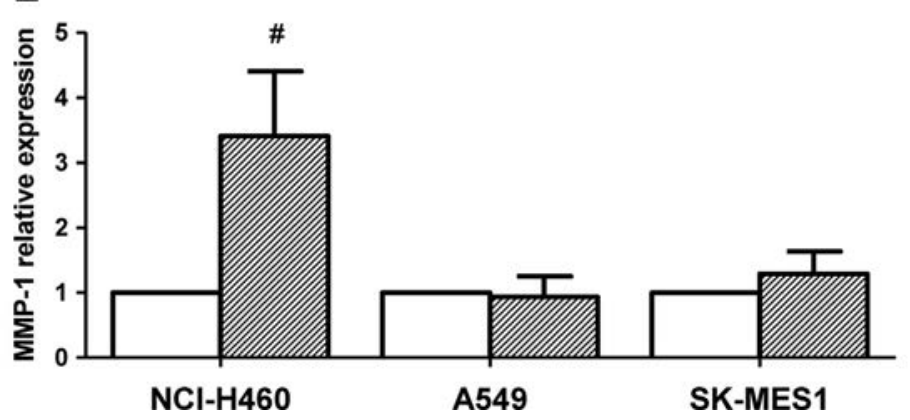

B

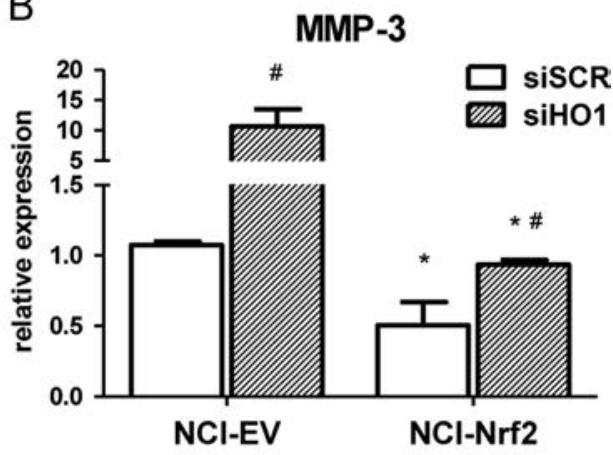

D

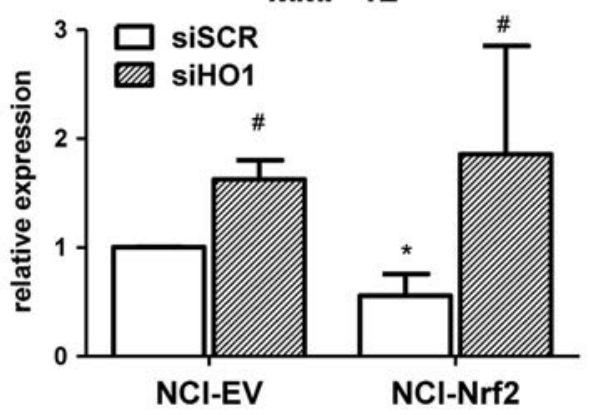

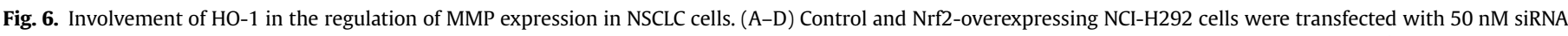

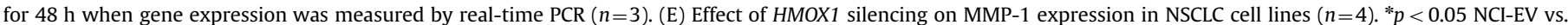
NCI-Nrf2, \#p $<0.05$ siSCR vs. siHO1.

transcription factor Nrf2 exerts antitumoral effects, attenuating tumor cell proliferation, migration, and expression of matrix metalloproteinases. Its actions are largely attributable to the principal ARE-regulated gene heme oxygenase-1, implying that the Nrf2HO-1 axis could be one of the major molecular pathways contributing to the relatively mild clinical presentations of lung MECs.

Restoration of physiological levels of reactive oxygen species from oncogenic ones by antioxidant actions of Nrf2 target proteins has been associated with suppressed tumor growth in models of glioma, melanoma, and pancreatic cancer [21-23]. Nevertheless, since the treatment of the NCI-H292 cell line with an antioxidant, $\mathrm{N}$-acetylcysteine, resulted in enhanced tumor cell proliferation (data not shown), the effects of Nrf2 overexpression in our model of MEC could rather be redox-independent. Our data suggest that microRNAs may be involved in modulation of MEC cell proliferation, as Nrf2-overexpressing cells exhibited enhanced expression of the tumor suppressor miRNAs miR-193b, miR-181a, and miR424 and a decreased level of oncogenic miR-378, which we and others have previously shown to play a protumorigenic role in lung NSCLC $[9,24]$. Down-regulation of miR-378 and attenuation of cell proliferation and migration in Nrf2-overexpressing MEC cells was reversed by HMOX1 knockdown, establishing this Nrf2 target gene as a central mediator of its action. This finding could be explained by recent data of Biswas et al. showing that a nuclear form of HO-1 interacts with Nrf2 and stabilizes it, thereby reinforcing its action [25]. Not surprisingly, a key role of HO-1 in mediating the effects of Nrf2 has been also reported for human oral squamous cell carcinoma cells in a model of mollugin-induced growth inhibition and apoptosis [26] and in human breast cancer cells in induction of p53 by 15 -deoxy- $\Delta 12,14$-prostaglandin $\mathrm{J} 2$ [27]. The latter is in accordance with our recent observation of increased p53 levels in NCI-H292 cells overexpressing HMOX1 [9], additionally corroborated here by microarray data showing a strong decrease in the number of transcripts for Mdm2 protein, which is known to be a negative regulator of p53 protein stability. We have already demonstrated [9] that inhibition of MEC cell proliferation and migration by HO- 1 is replicated by treatment of cells with carbon monoxide.

Transcriptome analysis allowed us to reveal additional molecular pathways potentially involved in attenuation of tumorigenic potential of MEC cells by HO-1, including up-regulation of the known negative regulators of NSCLC growth GAS-1 [28] and p57 (CDKN1C) [29], down-regulation of c-myc protooncogene [30], and decreased expression of cyclin D1, which is overexpressed in 25- 

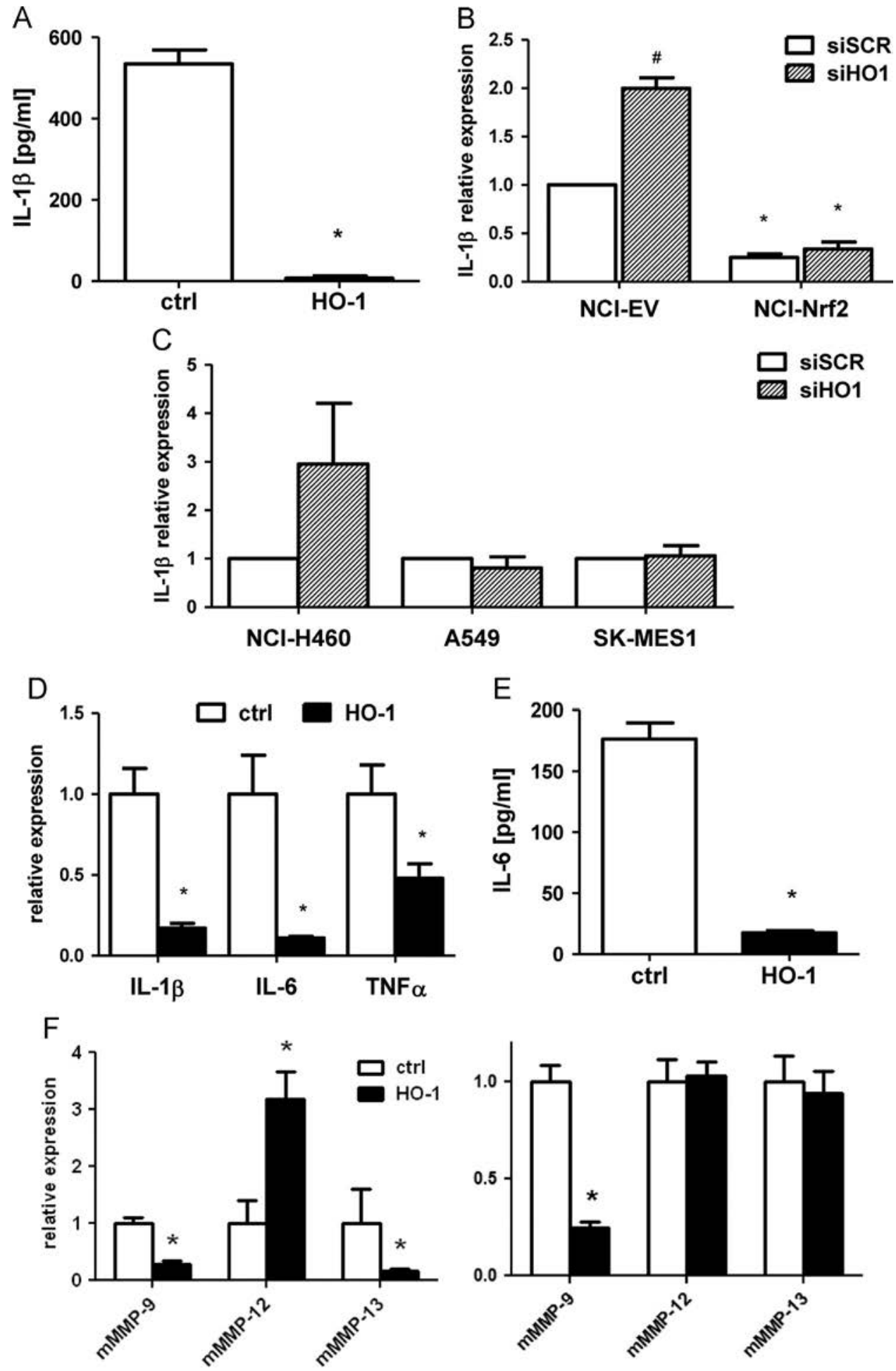

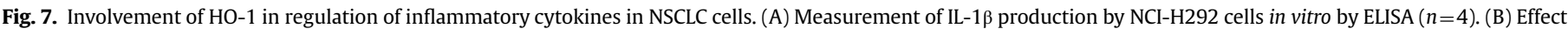

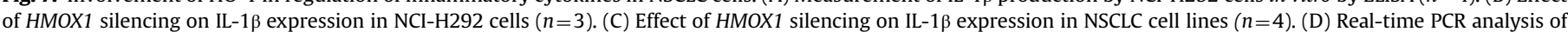

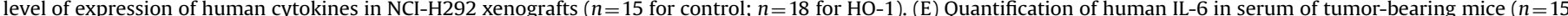

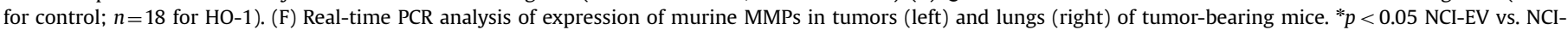
transgene, $\# p<0.05$ siSCR vs. siHO1.

$47 \%$ of clinical cases of NSCLC, correlating with bad prognosis [31].

We have previously shown, using another HMOX1-overexpressing $\mathrm{NCI}-\mathrm{H} 292$ cell line, that inhibition of MEC tumor growth in vivo is associated with decreased vascularization and oxygenation of cancer tissue [9]. Here we demonstrate that xenografting of the NCI-HO-1 cell line to NOD-SCID mice reveals significant attenuation of cancer cell metastatic potential. It coincides with down-regulation of matrix metalloproteinases including MMP-9, an enzyme down-regulated in low-grade lung MEC specimens [12] that has been already reported to be negatively regulated by HO-1 in breast cancer cells $[32,33]$. Nevertheless, MMP-9 was not inhibited by Nrf2 overexpression, while the 
general profile of MMP expression of NCI-Nrf2 cells not only mimicked that of $\mathrm{NCI}-\mathrm{HO}-1$ cells, but also displayed attenuated mRNA levels of additional enzymes. This broad inhibitory effect could be attributed to a general modulation of cellular signaling by altered redox status, as it has already been demonstrated that in prostate cancer cells a more reducing intracellular environment is associated with down-regulation of MMPs and decreased cell invasiveness [34], and we have previously reported that HMOX1overexpressing cells exhibit reduced levels of intracellular reactive oxygen species [9]. However, putatively redox-independent actions of Nrf2 target proteins may also be of importance, as exemplified in our study by the increased levels of MMPs in Nrf2overexpressing cells following $\mathrm{HO}-1$ knockdown. The potential mechanism of direct regulation of MMP expression by HO-1 requires further study. It may involve heme cleavage products such as CO, biliverdin, and bilirubin, which have been previously reported to attenuate activity of ERK1/2 kinases, diminishing nuclear translocation of the transcription factors AP- 1 and NF- $\kappa B$, and thus inhibiting transcription of MMPs [35,36]. Our findings of the key role of HO-1 in modulating the expression of MMPs in NCI-H292 are further corroborated by a uniform up-regulation of all the examined MMPs in control cells treated with siRNA against HO-1, which exhibited the lowest expression of HO-1 among the cell lines under study. Given the relatively low basal expression of HO1 in NCI-H292 cells as compared with other NSCLC models [9], this suggests that even a minimal level of HO-1 induction in MECs would be sufficient for suppression of expression of numerous matrix-degrading enzymes, possibly contributing to the relatively mild clinical phenotypes of these tumors.

The strongest effect of HMOX1 knockdown was observed for MMP-1 and MMP-3, the two proteins that have been reported to be regulated by interleukin-1 $\beta$ in A549 lung adenocarcinoma [18]. Indeed, silencing of HMOX1 in NCI-EV cells caused a significant upregulation of IL- $1 \beta$, while it had no effect on the inhibition of IL- $1 \beta$ expression in Nrf2-overexpressing cells, suggesting that the modulation of expression of the inflammatory cytokine could be involved in the action of Nrf2-HO-1 axis in regulating MMPs. Carbon monoxide, identified by our previous study as the major mediator of HO- 1 action in the MEC cells [9], is known to attenuate production [37] and signaling [38] of interleukin-1.

Consistent with the above, HMOX1-overexpressing cells produced very low amounts of IL- $1 \beta$ in vitro. $\mathrm{NCI}-\mathrm{HO}-1$ xenografts in NOD-SCID mice exhibited inhibited expression of IL-1 $\beta$, TNF $\alpha$, and IL-6, the last of which could be also detected at decreased levels in the blood of tumor-bearing animals. This anti-inflammatory effect coincided with down-regulation of murine MMP-9 and MMP-13 in the tumors and mMMP-9 in the lungs of xenografted animals, showing that the attenuation of the metastatic potential of MEC cells by HO-1 may be also attributed to the modulation of the tumor microenvironment and metastatic niche.

Taken together, we show the unique molecular consequences of activation of Nrf2-HO-1 axis in lung mucoepidermoid carcinoma that are associated with tumor-suppressive phenotype involving strong down-regulation of matrix metalloproteinases, which may explain clinical features of this rare subtype of non-small-cell lung cancer.

\section{Acknowledgments}

This work was supported by National Science Center Grants OPUS NCN 2012/07/B/NZ1/02881 (JD), HARMONIA NCN 2012/06/ $\mathrm{M} / \mathrm{NZ1} / 00008$ and N N301 460938 (AJ). We used also equipment obtained from EU Framework Programs POIG 02.01.00-12-064/08 and 02.02.00-00-014/08. UF was a recipient of a START Scholarship of the Foundation for Polish Science (FNP). KS is a recipient of a START Scholarship of the FNP. KS and UF are recipients of the Polish Ministry of Science and Higher Education's scholarship for young scientists. This research was conducted in the scope of the MiR-TANGo International Associated Laboratory (LIA). The Department of Medical Biotechnology was involved in the European Network on Gasotransmitters (COST Action BM1005). The Faculty of Biochemistry, Biophysics, and Biotechnology of Jagiellonian University is a partner of the Leading National Research Center (KNOW) supported by the Ministry of Science and Higher Education. The funders had no role in study design, data collection and analysis, decision to publish, or preparation of the manuscript.

\section{References}

[1] Y.S. Keum, B.Y. Choi, Molecular and chemical regulation of the Keap1-Nrf2 signaling pathway, Molecules 19 (7) (2014) 10074-10089.

[2] Slocum, S.L. and T.W.Kensler, Nrf2: control of sensitivity to carcinogens. Arch Toxicol. 85 (4): p. 273-284.

[3] C.R. Zhao, Z.H. Gao, X.J. Qu, Nrf2-ARE signaling pathway and natural products for cancer chemoprevention, Cancer Epidemiol 34 (5) (2010) 523-533.

[4] M. Xiang, et al., Nrf2: bane or blessing in cancer? J Cancer Res Clin Oncol 140 (8) (2014) 1251-1259.

[5] H.K. Na, Y.J. Surh, Oncogenic potential of Nrf2 and its principal target protein heme oxygenase-1, Free Radic Biol Med 67 (2014) 353-365.

[6] A.K. Bauer, T. Hill, 3rd, C.M. Alexander, The involvement of NRF2 in lung cancer, Oxid Med Cell Longev (2013) 7464322013.

[7] L.M. Solis, et al., Nrf2 and Keap1 abnormalities in non-small cell lung carcinoma and association with clinicopathologic features, Clin Cancer Res 16 (14) (2010) 3743-3753.

[8] J.R. Tsai, et al., High expression of heme oxygenase-1 is associated with tumor invasiveness and poor clinical outcome in non-small cell lung cancer patients, Cell Oncol (Dordr) 35 (6) (2012) 461-471.

[9] K. Skrzypek, et al., Interplay Between Heme Oxygenase-1 and miR-378 Affects Non-Small Cell Lung Carcinoma Growth, Vascularization, and Metastasis, Antioxid Redox Signal 19 (7) (2013) 644-660.

[10] A.C. Roden, et al., Histopathologic, immunophenotypic and cytogenetic features of pulmonary mucoepidermoid carcinoma, Mod Pathol 27 (11) (2014) 1479-1488.

[11] C.H. Chin, et al., Prognostic factors of tracheobronchial mucoepidermoid carcinoma-15 years experience, Respirology 13 (2) (2008) 275-280.

[12] J. Fan, et al., Comparative expression of matrix metalloproteinases in lowgrade mucoepidermoid carcinoma and typical lung cancer, Oncol Lett 2 (6) (2011) 1269-1273.

[13] E. Hadler-Olsen, J.O. Winberg, L. Uhlin-Hansen, Matrix metalloproteinases in cancer: their value as diagnostic and prognostic markers and therapeutic targets, Tumour Biol 34 (4) (2013) 2041-2051.

[14] M. Tertil, et al., Regulation and novel action of thymidine phosphorylase in non-small cell lung cancer: crosstalk with Nrf2 and HO-1, PLoS One 9 (5) (2014) e97070.

[15] A. Loboda, et al., HIF-1 induction attenuates Nrf2-dependent IL-8 expression in human endothelial cells, Antioxid Redox Signal 11 (7) (2009) 1501-1517.

[16] U. Florczyk, et al., Nrf2 regulates angiogenesis: effect on endothelial cells, bone marrow-derived proangiogenic cells and hind limb ischemia, Antioxid Redox Signal 20 (11) (2014) 1693-1708.

[17] M. Li, et al., Prognostic significance of matrix metalloproteinase-1 levels in peripheral plasma and tumour tissues of lung cancer patients, Lung Cancer 69 (3) (2010) 341-347.

[18] B.L. Petrella, D.A. Armstrong, M.P. Vincenti, Interleukin-1 beta and transforming growth factor-beta 3 cooperate to activate matrix metalloproteinase expression and invasiveness in A549 lung adenocarcinoma cells, Cancer Lett 325 (2) (2012) 220-226.

[19] A. Singh, et al., RNAi-mediated silencing of nuclear factor erythroid-2-related factor 2 gene expression in non-small cell lung cancer inhibits tumor growth and increases efficacy of chemotherapy, Cancer Res 68 (19) (2008) 7975-7984.

[20] S. Homma, et al., Nrf2 enhances cell proliferation and resistance to anticancer drugs in human lung cancer, Clin Cancer Res 15 (10) (2009) 3423-3432.

[21] A.K. Chakraborty, et al., Intracellular glutathione and its metabolizing enzyme activities in a metastatic variant melanoma cell line, Melanoma Res 2 (5-6) (1992) 315-319.

[22] J. Liu, et al., Redox regulation of pancreatic cancer cell growth: role of 
glutathione peroxidase in the suppression of the malignant phenotype, Hum Gene Ther 15 (3) (2004) 239-250.

[23] Y. Zhang, et al., Overexpression of copper zinc superoxide dismutase suppresses human glioma cell growth, Cancer Res 62 (4) (2002) 1205-1212.

[24] L.T. Chen, et al., MicroRNA-378 is associated with non-small cell lung cancer brain metastasis by promoting cell migration, invasion and tumor angiogenesis, Med Oncol 29 (3) (2012) 1673-1680.

[25] C. Biswas, et al., Nuclear Heme Oxygenase-1 (HO-1) Modulates Subcellular Distribution and Activation of Nrf2, Impacting Metabolic and Anti-oxidant Defenses, J Biol Chem 289 (39) (2014) 26882-26894.

[26] Y.M. Lee, et al., Involvement of Nrf2-mediated upregulation of heme oxygenase-1 in mollugin-induced growth inhibition and apoptosis in human oral cancer cells, Biomed Res Int (2013) 2106042013.

[27] D.H. Kim, et al., 15-deoxy-Delta12,14-prostaglandin J(2) induces p53 expression through Nrf2-mediated upregulation of heme oxygenase-1 in human breast cancer cells, Free Radic Res 48 (9) (2014) 1018-1027.

[28] A. Evdokiou, P.A. Cowled, Tumor-suppressive activity of the growth arrestspecific gene GAS1 in human tumor cell lines, Int J Cancer 75 (4) (1998) 568-577.

[29] I.S. Pateras, et al., Downregulation of the KIP family members p27(KIP1) and p57(KIP2) by SKP2 and the role of methylation in p57(KIP2) inactivation in nonsmall cell lung cancer, Int J Cancer 119 (11) (2006) 2546-2556.

[30] L.A. Robinson, et al., c-myc antisense oligodeoxyribonucleotides inhibit proliferation of non-small cell lung cancer, Ann Thorac Surg 60 (6) (1995) 1583-1591.
[31] D.C. Betticher, et al., Prognostic significance of CCND1 (cyclin D1) overexpression in primary resected non-small-cell lung cancer, Br J Cancer 73 (3) (1996) 294-300.

[32] C. Wang, et al., BMP-6 inhibits MMP-9 expression by regulating heme oxygenase-1 in MCF-7 breast cancer cells. J Cancer Res Clin Oncol 137 (6) (2011) 985-995.

[33] C.W. Lin, et al., Heme oxygenase-1 inhibits breast cancer invasion via suppressing the expression of matrix metalloproteinase-9, Mol Cancer Ther 7 (5) (2008) 1195-1206.

[34] L. Chaiswing, et al., Regulation of prostate cancer cell invasion by modulation of extra- and intracellular redox balance, Free Radic Biol Med 52 (2) (2012) $452-461$.

[35] S.W. Ryter, L.E. Otterbein, Carbon monoxide in biology and medicine, Bioessays 26 (3) (2004) 270-280.

[36] S.K. Moon, B.Y. Cha, C.H. Kim, ERK1/2 mediates TNF-alpha-induced matrix metalloproteinase-9 expression in human vascular smooth muscle cells via the regulation of NF-kappaB and AP-1: Involvement of the ras dependent pathway, J Cell Physiol 198 (3) (2004) 417-427.

[37] T. Takagi, et al., Inhalation of carbon monoxide ameliorates collagen-induced arthritis in mice and regulates the articular expression of IL-1beta and MCP-1, Inflammation 32 (2) (2009) 83-88.

[38] I. Garcia-Arnandis, et al., Control of cell migration and inflammatory mediators production by CORM-2 in osteoarthritic synoviocytes, PLoS One 6 (9) (2011) e24591. 